psychopraxis. neuropraxis

https://doi.org/10.1007/s00739-021-00696-2

Angenommen: 5. Januar 2021

(c) Der/die Autor(en) 2021

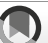

Dagmar Steinmair ${ }^{1}$ Tamara Prevendar ${ }^{2}$ Branca Milic $^{1,2} \cdot$ Marian Hochgerner $^{1,2}$. Henriette Löffler-Stastka ${ }^{1,2}$

${ }^{1}$ Klinik für Psychoanalyse und Psychotherapie, Medizinische Universität Wien, Wien, Österreich

${ }^{2}$ European Network on Individualized Psychotherapy Treatment of Young People with Mental Disorders (TREATme), Brüssel, Belgien

\title{
Psychotherapie-Outcome- Studien bei Psychosen, Depressionen und Persönlichkeitsstörungen im Jugend- und jungen Erwachsenenalter
}

ter zwischen 17,6 und 23,9\% angegeben [7-9].

Stigmatisierung, unzureichende Versorgungssituation, mangelnder Einsatz evidenzbasierter Therapiemethoden und Präventionsstrategien tragen dazu bei, dass eine Therapie häufig spät, unzureichend und nicht individualisiert passiert. Psychische Störungen im Jugendalter sind oft der Beginn einer lebenslangen Patientenkarriere mit entsprechend hohen Kosten (privat und öffentlich) anstatt eines Recovery mit der Möglichkeit Selbstbestimmtheit zu erlangen.

Dieser Artikel ist Teil des TREATme Projekts des European Network on Individualized Psychotherapy Treatment of Young People with Mental Disorders (Registrierung: PROSPERO 2020 CRD42020166756): Systematische Literaturrecherchen zu Therapieoptionen bei Depression, Persönlichkeitsstörungen und psychotischen Störungen befassen sich mit der Frage existierender Evidenz für die Effektivität psychotherapeutischer Methoden bei diesen Erkrankungen in der Adoleszenz.

\section{Fallbeispiel: Polypharmazie bei Borderline-Persönlichkeits- störung (BPD)}

Madan et al. schildern die Hospitalisierung von Frau A., einer Unternehmerin mit BPD, welche im mittleren Alter, aufgrund einer schweren Depression mit persistierenden suizidalen Gedanken, schwerer Angststörung sowie Somatisierung zur Aufnahme kommt [10]. Die Patientin zeigte sich somnolent, es bestand eine Polypharmazie mit schlechter Compliance und Diabetes-mellitusKontrolle sowie ein Reizdarmsyndrom. Erste behandlungsbedürftige psychische Symptome waren im Alter von 4 Jahren dokumentiert, der Facharzt für Kinder- und Jugendpsychiatrie hatte Amphetamine bei Hyperaktivität verordnet. Im Teenageralter mündete eine 7-jährige Phase mit suizidalen Gedanken in einen Suizidversuch, welcher mit 19 zur ersten psychiatrischen Hospitalisierung führte. Die Anamnese umfasste selbstverletzende Handlungen, Risikoverhalten sowie emotionale Instabilität, Stimmungsreaktivität, ein Gefühl der Leere, Trennungsängste, eingeschränkte Impulskontrolle und eine Binge-Eating-Störung. Frau A. war bis zum Zeitpunkt der aktuellen Hospitalisierung bei 4 Psycho- 
therapeuten und 3 Fachärzten für Psychiatrie in Behandlung. Zuletzt kam es infolge Suizidalität zu einem Therapeutenwechsel nach einer 6 Jahre dauernden Therapie. Den ersten psychischen Symptomen war der Tod des Vaters vorangegangen, die Mutter heiratete 3-mal neue Partner, Bezugsperson war eine Nanny. Frau A. lebte sozial zurückgezogen, nachdem ihr langjähriger Ehemann unerwartet verstorben war. Finanziell hatte sie sich in der Zeit vor der aktuellen Hospitalisierung in eine prekäre Situation gebracht.

Die aktuelle stationäre Intervention umfasste während 57 Behandlungstagen vor allem den Aufbau einer stabilen Therapeuten-Patient-Beziehung und ein Training für den Umgang mit Distress (Hilfesuchverhalten, Emotionsregulierung und Kontrolle, Mentalisierung) sowie eine systematische Neueinstellung der Pharmakotherapie [10]. Interventionen umfassten neben der supportiven und expressiven Psychotherapie im Einzelsetting (kognitive Verhaltenstherapie, $2 \times$ /Woche), rehabilitative Gruppentherapie $(2-4 \times$ /Woche) und Interventionen im Milieu/der Familie (Sozialarbeit, $1 \times /$ Woche).

\section{Diskussion}

Das Fallbeispiel zeigt den fluktuierenden Verlauf einer Persönlichkeitsstörung mit Wurzeln und prodromaler Phase im Kindes- und Teenageralter bis hin zur Dekompensation, mit Komorbiditäten im frühen Erwachsenenalter, welche im ambulanten Setting nicht therapierbar war, wobei trotz Suizidalität erst verzögert eine stationäre Aufnahme erfolgte. Details $\mathrm{zu}$ den psychotherapeutischen Behandlungen im Vorfeld dieses ungünstigen Verlaufs fehlen - allerdings wird klar, dass vor dem 19. Lebensjahr keine Psychotherapie stattfand. Weiters ist ersichtlich, dass es zu wiederholten stationären Kurzaufenthalten, zu kurzfristigen Interventionen durch unterschiedliche Behandler und $\mathrm{zu}$ einer Akkumulation von Pharmaka kam anstatt zu einer koordinierten und frühen Intervention mit Rücksicht auf den komplexen Langzeitverlauf, welcher bei symptomatischen Episoden mit prolongierter Dauer zu erwarten war.

\section{Behandlungsmöglichkeiten bei Persönlichkeitsstörungen}

Mit $1 \%$ Prävalenz in der jugendlichen Gesamtbevölkerung ist die BPD insgesamt selten, allerdings wird die Diagnose in psychiatrischen Settings an bis zu 50\% der Patienten vergeben [11], der Erkrankungsgipfel liegt in der späten Adoleszenz, mit einer Remission auf Symptomebene nach 10 Jahren von über 85-99\% bei weiter eingeschränktem psychosozialen Funktionsniveau [11]. Komorbiditäten und Leidensdruck bei Patienten mit Persönlichkeitsstörung sind hoch - die Lebenserwartung ist deutlich reduziert.

Nach heutigem Wissensstand konsolidiert sich die Persönlichkeitsstruktur über Jahrzehnte langsam und mehrere psychotherapeutische Ansätze bieten vielversprechende Behandlungsoptionen, sodass erste Schritte in Richtung Entstigmatisierung geschafft sind.

\section{》) Evidenz aus RCTs für die Wirksamkeit der Psychotherapie bei BPD}

Die systematische Literaturrecherche der TREATme-Gruppe (Registration: PROSPERO 2020 CRD42020166756) bestätigte die Evidenz aus randomisiert kontrollierten Studien (RCTs) bei BPD für die Wirksamkeit der kognitiven Verhaltenstherapie sowie der kognitiv analytischen Therapie. Vielversprechende Ergebnisse zeigten RCTs zur übertragungsfokussierten Psychotherapie. Für aggressiv-oppositionelles Verhalten und Patienten mit antisozialen Persönlichkeitsmerkmalen gibt es einen Wirksamkeitsnachweis für die Mode Deactivation Therapy. Psychoedukation und emotionsregulierende Therapien zeigten in jeweils einer RCT bei BPD einen positiven Einfluss auf die Symptomatik. Ergebnisse aus Kohortenstudien zur mentalisierungsbasierten Therapie, psychodynamischen Therapie und kognitiven Schematherapie im Gruppensetting zeigten einen symptomlindernden Effekt.

Es gelang bislang nicht, RCTs mit ausreichend großer Stichprobe und einheitlicher Methodik sowie Langzeit-Follow-
Up zu generieren, ähnlich in den verschiedenen Ländern weltweit. Das lässt viele Fragen hinsichtlich der Wirkungsweise von Psychotherapie offen, zumal auch für existierende Ergebnisse die Replikation aussteht. Es bleibt daher eine sehr individualisierte Vorgehensweise empfehlenswert, möglichst höherfrequent und unter Einbezug des sozialen Milieus. Bislang lag der Fokus der meisten RCTs auf der BPD, für die Therapie anderer Persönlichkeitsstörungen in der Adoleszenz gibt es kaum Literatur.

\section{Fallserie: Prodromale Phase bei Psychosen im Jugendalter}

Ching et al. beobachteten 10 Patienten, welche im Rahmen des EASY (Early Assessment Service for Young People with Psychosis)-Programms in Hongkong identifiziert worden waren, mit möglichen Prodromi einer Psychose [12]. Die Probanden erhielten psychotherapeutisches Case-Management und ggf. Psychopharmaka. Die Fälle mit prodromalen Anzeichen für eine Psychose wurden nach den Kriterien von McGorry et al. auf einem Kontinuum von gesund bis manifest psychotisch klassifiziert [13]. Die Verläufe wurden in gesamt 4 Fällen als fluktuierend, in 3 als gebessert, in 2 als unverändert und in einem Patienten als Übergang in eine Psychose klassifiziert.

Fallvignette: (aus [12]) Der 20-jährige Patient J. war zum Zeitpunkt der Erstmanifestation bereits seit 2 Jahren als mutistisch und affektflach aufgefallen, zudem zeigte er graduell zunehmende Auffälligkeiten des Verhaltens (Polydipsie, Nudismus - zusätzlich wurde die Kleidung auf die Straße geworfen, Bewegungsarmut). Nach 6 Monaten wurden Antipsychotika verordnet - die Symptomatik fluktuierte weiter.

Bei Patienten mit stabiler Verbesserung vs. Patienten mit Fluktuation wurde bei letzteren Distress aufgrund (akustischer) Halluzinationen oder Wahnvorstellungen und Komorbidität mit Depression und Angsterkrankungen beobachtet, wobei sich die Gruppen hinsichtlich Beginn, Ausprägung und Konstanz dieser Symptomatik unterschieden. Ungünstig auf den Verlauf wirkten sich kriti- 
sche Lebensereignisse und soziale Isolation aus, günstig waren ein stützendes Umfeld und vorhandene Krankheitseinsicht. Nur ein Patient entwickelte eine manifeste Psychose, dieser hatte eine positive Familienanamnese sowie bereits seit 2 Jahren Wahnvorstellungen.

\section{Diskussion}

Die oben genannte Fallserie [12] illustriert, dass sich zwar psychotische Erkrankungen Jahre vor der Diagnosestellung ankündigen können, dass sich jedoch Verdachtsfälle nicht stets zum Vollbild entwickeln müssen. Frühe Interventionen führten in dieser Risikogruppe wahrscheinlich in 9 von 10 Fällen zur Verhinderung einer Psychose - allerdings fehlt eine Kontrollgruppe.

\section{) Schwierigkeit liegt in der Früherkennung von Risikopatienten}

Auf den Einsatz präventiver Strategien in Risikopopulationen setzte auch eine Untersuchung an der University of Oxford, in welcher Interventionen in 12 „Ultrahigh-risk“-Fällen auf der Hypothese basierten, dass allein die psychotherapeutische Behandlung von Schlafstörungen eine Verbesserung im negativen Affekt und der psychotischen Symptome bringt; Patienten mit organischer Ursache für die Schlafstörung waren ausgeschlossen [14].

Für den Einsatz effektiver Interventionen liegt die Schwierigkeit in der Früherkennung von Risikopatienten. Entscheidend ist zudem das Erkennen jener Fälle, bei denen organische Ursachen die Symptomatik bedingen. Ching et al. stellen keine differentialdiagnostischen Überlegungen an - allerdings erfolgte eine $\mathrm{Ab}$ klärung der Probanden mittels „Structured Clinical Interview for DSM Disorders" und eine monatliche Kontrolle an einer psychiatrischen Ambulanz [12].
Psychosen - existierende Evidenz für den frühen Einsatz von Psychotherapie

Die Beiträge, welche die Einschlusskriterien der TREATme-Gruppe erfüllten (26 Studien), verglichen bis auf eine Studie die untersuchte Therapiemethode mit einer Kontrollgruppe. Die meiste Evidenz gibt es bislang für die (kognitive) Verhaltenstherapie (9 von 26) und die kognitive Remediation-Therapie (11 von 26).

Für die Verhaltenstherapie zeigte sich zwar eine Besserung auf Symptomebene sowie auch der Angst, Depressivität und allgemeinen Psychopathologie, allerdings nicht signifikant besser als in der Kontrollgruppe. Ein signifikanter Wirksamkeitsnachweis wurde für die kognitive Remediation-Therapie hinsichtlich Verbesserung der Positivsymptomatik und Depressivität erbracht. Frühe Interventionen, strukturierte Gruppenintervention und Psychoedukation sowie ein „integrated care program“ wirkten sich signifikant positiv auf das psychosoziale Funktionsniveau aus.

Zusammenfassend lässt sich sagen, dass für psychotische Patienten, unabhängig von der Psychotherapieschule, methodisch einheitliche RCTs mit ausreichend großer Fallzahl und Dauer fehlen. Unabhängig von der Wahl der Psychotherapiemethode weist die Evidenz jedoch darauf hin, dass ein frühzeitiger Einsatz von Psychotherapie wirksam sein kann, inkl. Case-Management und Interventionen, welche das Milieu und die Ressourcen des Patienten berücksichtigen.

\section{Depression}

Im Fall von Frau A. kam zur BPD-Diagnose noch die Komorbidität der Depression bis hin zur chronisch rezidivierenden Suizidalität mit Akkumulation diverser Psychopharmaka [10].

Eine im Lancet publizierte Metaanalyse zeigt, trotz eingeschränkter Verfügbarkeit von qualitativ hochwertiger Evidenz, für Fluoxetin das beste Profil hinsichtlich Effektivität und Akzeptabilität bei Jugendlichen mit akuter mittelschwerer depressiver Erkrankung, gefolgt von einer Kombination von Fluoxetin mit psychopraxis. neuropraxis

https://doi.org/10.1007/s00739-021-00696-2

(c) Der/die Autor(en) 2021

D. Steinmair - T. Prevendar - B. Milic . M. Hochgerner $\cdot$ H. Löffler-Stastka

Psychotherapie-OutcomeStudien bei Psychosen, Depressionen und Persönlichkeitsstörungen im Jugend- und jungen Erwachsenenalter

\section{Zusammenfassung}

Bis zu $50 \%$ aller Jugendlichen entwickeln psychische Symptome, ein Viertel wird psychisch krank. Die Hälfte der psychisch erkrankten Erwachsenen war bereits im Jugendalter von einer psychischen Störung betroffen. Die aufkommende Evidenz für den positiven Effekt früher psychotherapeutischer Interventionen macht Screeningmaßnahmen und begleitende Forschung notwendig.

Schlüsselwörter

Psychische Erkrankungen · Adoleszenz · Prävention - Wirksamkeit - Evidenzbasierte Präzision

\section{Psychotherapy Outcome Studies for Psychosis, Depression and Personality Disorders in Young People}

\section{Abstract}

Up to $50 \%$ of all young people develop psychological symptoms; one-quarter become mentally ill. Half of mentally ill adults were affected by a mental disorder in their youth. Arising evidence for the positive effect of early psychotherapeutic interventions necessitate screening strategies and differentiated research.

Keywords

Mental disorders - Adolescence · Prevention . Efficacy · Evidence-based precision

Psychotherapie (Verhaltenstherapie, psychodynamische Therapie), welche besonders bei Suizidalität gute Ergebnisse zeigte [15]. Für andere Psychopharmaka, welche bei Erwachsenen wirksamer als Placebo waren, konnte kein Wirksamkeitsnachweis in dieser Altersgruppe dokumentiert werden [15]. Für Venlafaxin etwa wurde in der erwähnten Metaanalyse erneut ein erhöhtes Risiko gefunden 
und für andere Pharmaka ist die Datenlage diesbezüglich nicht ausreichend [15]. Gerade zu Beginn einer Psychotherapie sollte im Falle des Einsatzes von Antidepressiva bei Jugendlichen ein Monitoring hinsichtlich erhöhter Suizidalität erfolgen und der additive Effekt von Psycho- und Pharmakotherapie bedacht werden. Für die Musiktherapie konnte ein augmentativer Effekt gezeigt werden, wenn sie mit Verhaltenstherapie kombiniert wird [16].

\section{Fazit für die Praxis}

- Die Beiträge zur Recherche waren trotz hoher klinischer Relevanz limitiert, dies vorwiegend aufgrund der heterogenen Methodik, kleiner Fallzahlen, fehlender Kontrollgruppen - dennoch nimmt die Evidenz für positive Effekte früher psychotherapeutischer Interventionen zu.

- Psychotherapeutische Interventionen im Jugendalter sollten deshalb niederschwellig und früh erfolgen können, nach Ausschluss somatischer Ursachen.

- Frühe Symptome sind häufig unspezifisch. Herausfordernd ist das Herausfiltern jener adoleszenten Patienten mit psychischen Symptomen mit später chronischem, komplizierten Verlauf.

- Die unkritische Behandlung von Symptomen mit Psychopharmaka kann zu Sekundärkomplikationen infolge Polypharmazie führen. Bei depressiver Symptomatik besteht ein erhöhtes Suizidrisiko am Beginn einer Therapie mit Antidepressiva, ein Monitoring ist notwendig.

- Die Versorgungssituation ist als unzureichend einzustufen, die Stigmatisierung von psychischen Erkrankungen erhöht zudem die Hemmschwelle Hilfe in Anspruch zu nehmen.

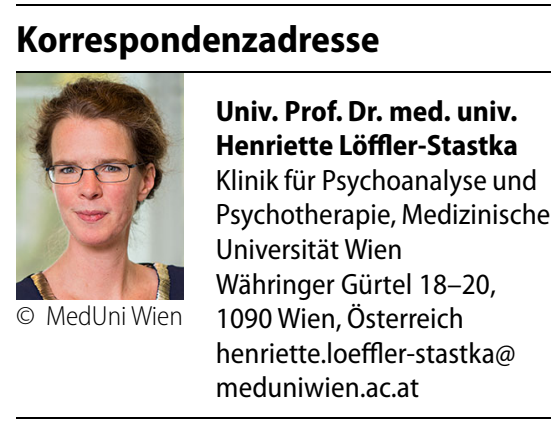

Danksagung. Die Autoren danken den Workinggroup-I-Mitgliedern der TREATme-Gruppe https:// www.treat-me.eu/ für die konstruktive Zusammenarbeit.

Funding. European Cooperation in Science and Technology (COST).

Funding. Open access funding provided by Medical University of Vienna.

\section{Einhaltung ethischer Richtlinien}

Interessenkonflikt. D. Steinmair, T. Prevendar, B. Milic, M. Hochgerner und H. Löffler-Stastka geben an, dass kein Interessenkonflikt besteht.

Für diesen Beitrag wurden von den Autoren keine Studien an Menschen oder Tieren durchgeführt. Für die aufgeführten Studien gelten die jeweils dort angegebenen ethischen Richtlinien.

Open Access. Dieser Artikel wird unter der Creative Commons Namensnennung 4.0 International Lizenz veröffentlicht, welche die Nutzung, Vervielfältigung Bearbeitung, Verbreitung und Wiedergabe in jeglichem Medium und Format erlaubt, sofern Sie den/die ursprünglichen Autor(en) und die Quelle ordnungsgemäß nennen, einen Link zur Creative Commons Lizenz beifügen und angeben, ob Änderungen vorgenommen wurden.

Die in diesem Artikel enthaltenen Bilder und sonstiges Drittmaterial unterliegen ebenfalls der genannten Creative Commons Lizenz, sofern sich aus der Abbildungslegende nichts anderes ergibt. Sofern das betreffende Material nicht unter der genannten Creative Commons Lizenz steht und die betreffende Handlung nicht nach gesetzlichen Vorschriften erlaubt ist, ist für die oben aufgeführten Weiterverwendungen des $\mathrm{Ma}$ terials die Einwilligung des jeweiligen Rechteinhabers einzuholen.

Weitere Details zur Lizenz entnehmen Sie bitte der Lizenzinformation auf http://creativecommons.org/ licenses/by/4.0/deed.de.

\section{Literatur}

1. Patton GC, Coffey C, Romaniuk H, Mackinnon A, Carlin JB, Degenhardt L, Olsson CA, Moran P (2014) The prognosis of common mental disorders in adolescents: a 14-year prospective cohort study. Lancet 383(9926):1404-1411. https://doi.org/10. 1016/S0140-6736(13)62116-9
2. Kessler RC, Avenevoli S, Costello EJ, Georgiades K, Green JG, Gruber MJ, He JP, Koretz D, McLaughlin KA, Petukhova M, Sampson NA, Zaslavsky AM, Merikangas KR (2012) Prevalence, persistence, and sociodemographic correlates of DSM-IV disorders in the national comorbidity survey replication adolescent supplement. Arch Gen Psychiatry 69(4):372-380. https://doi.org/10. 1001/archgenpsychiatry.2011.160

3. Fuchs $M$, Bösch $A$, Hausmann $A$, Steiner $H$ (2013) "The child is father of the man“: review von relevanten Studien zur Epidemiologie in der kinder- und Jugendpsychiatrie. Z Kinder Jugendpsychiatr Psychother 41(1):45-55

4. Fuchs M, Karwautz A (2017) Epidemiologie psychischer Störungen bei Kindern und Jugendlichen. Neuropsychiatr 31:96-102. https://doi.org/10. 1007/s40211-017-0238-x

5. Merikangas KR, He JP, Burstein M, Swanson SA, Avenevoli S, Cui L, Benjet C, Georgiades K, Swendsen J (2010) Lifetime prevalence of mental disorders in U.S. adolescents: results from the national comorbidity survey replication-adolescent supplement (NCS-A). J Am Acad Child Adolesc Psychiatry 49(10):980-989. https://doi.org/10. 1016/j.jaac.2010.05.017

6. Ravens-Sieberer U, Wille N, Erhart M, Bettge S, Wittchen HU, Rothenberger A, Herpertz-Dahlmann B, Resch F, Hölling $H$, Bullinger $M$, Barkmann $C$, Schulte-Markwort M, Döpfner M, BELLA study group (2008) Prevalence of mental health problems among children and adolescents in Germany: results of the BELLA study within the national health interview and examination survey. EurChild Adolesc Psychiatry 17(1):22-33

7. Barkmann C, Schulte-Markwort M (2004) Prevalence of emotional and behavioral disorders in children and adolescents in Germany - a systematic literature review. Psychiatr Prax 31(6):278-287. https://doi.org/10.1055/s-2003-814855

8. Polanczyk GV, Salum GA, Sugaya LS, Caye A, Rohde LA (2015) Annual research review: a metaanalysis of the worldwide prevalence of mental disorders in children and adolescents. J Child Psychol Psychiatry Allied Discip 56(3):345-365. https://doi.org/10.1111/jcpp.12381

9. Wagner G, Zeiler M, Waldherr K, Philipp J, Truttmann S, Dür W, Treasure JL, Karwautz A (2017) Mental health problems in Austrian adolescents: a nationwide, two-stage epidemiological study applying DSM-5 criteria. Eur Child Adolesc Psychiatry 26(12):1483-1499. https://doi.org/10. 1007/s00787-017-0999-6

10. Madan A, Oldham JM, Gonzalez S, Fowler JC (2015) Reducing adverse polypharmacy in patients with borderline personality disorder: an empirical case study. Prim Care Companion CNS Disord. https:// doi.org/10.4088/PCC.14m01760

11. Kaess M, Brunner R, Chanen A (2014) Borderline personality disorder in adolescence. Pediatrics 134(4):782-793. https://doi.org/10.1542/peds. 2013-3677

12. Ching EY, Lee EH, Hui CL, Lin JX, Chang WC, Chan SK, Chen EY (2015) Prodromal psychosis: a case series of ten symptomatic patients. East Asian Arch Psychiatry 25(1):35-41

13. McGorry PD, Purcell R, Hickie IB, Yung AR, Pantelis C, Jackson HJ (2007) Clinical staging: a heuristic model for psychiatry and youth mental health. Med J Aust 187(S7):S40-S42

14. Bradley J, Freeman D, Chadwick E, Harvey AG, Mullins B, Johns L, Sheaves B, Lennox B, Broome M, Waite $F$ (2018) Treating sleep problems in young people at ultra-high risk of psychosis: a feasibility 
case series. Behav Cogn Psychother 46(3):276-291.

https://doi.org/10.1017/S1352465817000601

15. Zhou X, Teng T, Zhang Y, Del Giovane C,

Furukawa TA, Weisz JR, Li X, Cuijpers P, Coghill D,

Xiang Y, Hetrick SE, Leucht S, Qin M, Barth J,

Ravindran AV, Yang L, Curry J, Fan L, Silva SG,

Cipriani A, Xie P (2020) Comparative efficacy and acceptability of antidepressants, psychotherapies, and their combination for acute treatment of children and adolescents with depressive disorder: a systematic review and network meta-analysis. Lancet Psychiatry 7(7):581-601. https://doi.org/ 10.1016/S2215-0366(20)30137-1

16. Witusik A, Pietras T (2019) Music therapy as a complementary form of therapy for mental disorders. Pol MerkurLekarski 47(282):240-243

Hinweis des Verlags. Der Verlag bleibt in Hinblick auf geografische Zuordnungen und Gebietsbezeichnungen in veröffentlichten Karten und Instituts-

adressen neutral. 